\title{
Estudo de Adesivos Condutivos Isotrópicos Utilizados para a Fixação de Componentes Eletrônicos
}

Egont Alexandre Schenkel ${ }^{1}$, Talita Mazon ${ }^{1}$, Fernando Fujimoto ${ }^{1}$, Sebastião Eleutério Filho², Carlos Roberto Mendes de Oliveira², Marcos Batista Cotovia Pimentel ${ }^{2}$, Márcio Tarozzo Biasoli ${ }^{1}$

\author{
${ }^{1}$ Divisão de Empacotamento Eletrônico - DEE \\ e-mail: egont.schenkel@,cenpra.gov.br, talita.anselmo@,cenpra.gov.br, fernando.fujimoto@,cenpra.gov.br, \\ marcio.biasoli@cenpra.gov.br \\ ${ }^{2}$ Divisão de Qualificação e Análise de Produtos Eletrônicos - DAPE \\ Centro de Pesquisas Renato Archer - CenPRA Rod. D. Pedro I (SP 65), km 143,6 - Campinas - SP, \\ CEP13069-91 \\ e-mail: sebastiao.eleuterio@cenpra.gov.br, carlos.oliveira@cenpra.gov.br, marcos.pimentel@cenpra.gov.br.
}

\section{RESUMO}

Atualmente, os adesivos condutivos estão sendo visados como possível alternativa para substituir as pastas de solda convencionais na montagem de componentes eletrônicos em placas de circuito impresso (PCI), por serem menos nocivos ao meio ambiente e pela capacidade de fazer junções fine pitch. No entanto, pouco se conhece sobre o comportamento e propriedades dos adesivos condutivos isotrópicos, na forma de pasta, na montagem de componentes eletrônicos em PCIs. Este trabalho teve como objetivo estudar as propriedades físicas e químicas, assim como o comportamento de alguns adesivos condutivos comerciais, visando a sua utilização na "soldagem" de componentes eletrônicos. Três diferentes adesivos condutivos isotrópicos comerciais (na forma de pasta) foram utilizados. Inicialmente, filmes espessos destes adesivos foram preparados em substrato de vidro com a finalidade de analisar o comportamento de resistividade elétrica em função do tempo e da temperatura de cura. Em uma segunda etapa, os adesivos foram utilizados para fixar diferentes tipos de componentes eletrônicos em PCI. Com o intuito de caracterizar a microestrutura da interface componente/adesivo/placa, amostras foram caracterizadas por microscopia óptica e microscopia eletrônica de varredura. Para verificar a viabilidade do uso desses adesivos, foram feitos testes elétricos funcionais e os resultados obtidos foram comparados aos da solda convencional. Verificou-se que os adesivos condutivos apresentaram propriedades físicas compatíveis aos da pasta de solda convencional.

Palavras chaves: adesivos condutivos isotrópicos, soldas livres de chumbo, tecnologia de montagem em superfície.

\section{Study of Isotropic Conductive Adhesives for SMD Mounting}

\section{ABSTRACT}

Recently, conductive adhesives have been considered as alternatives for replacing conventional solders in the mount technology of the electronic components on print circuit board (PCI), due to necessity to look for environment - friendly materials and fine pitch components. However, there is a little information about the behavior and properties of the isotropic conductive adhesives (as paste) for using in the mounting of the electronic components. The objective of this work was to study the physical and chemical properties, as well as the behavior of isotropic conductive adhesives aiming their use in the "soldering" of electronic components. Three different commercial isotropic conductive adhesives were utilized in this study. Firstly, thick films were prepared on glass substrate aiming to analyze the behavior of resistivity as a function of the time and temperature of cure. Secondly, the adhesives were utilized for fixing different kinds of electronic components on PCI. Then, the samples were characterized by optical and scanning electronic microscopy with the purpose of verifing the component/adhesive/PCI interface. Electric tests were realized to verify the viability of the use of these adhesives and the results are compared with the conventional solders. It was observed that the isotropic conductive adhesives showed properties compatible to conventional paste solders.

Keywords: isotropic conductive adhesives, lead free solders, surface mount technology. 


\section{INTRODUÇÃO}

Atualmente, a indústria eletrônica tem procurado por materiais menos nocivos ao meio ambiente, movidos por diretivas como a RoHS (Restriction of Hazordous Substances) e WEEE (Waste Electrical and Electronics Equipments), publicadas nos países da União Européia e da Ásia, as quais proíbem o uso de algumas substâncias ou elementos tóxicos em produtos elétricos e eletrônicos a partir de 1 de julho de 2006 [1]. Nos Estados Unidos, a "Environmental Protection Agency" também estipulou que a porcentagem de descarte do chumbo no produto final deve ser menor que $0,1 \%$.

A preocupação da indústria eletrônica com estas diretivas está no fato da maioria delas utilizarem uma liga metálica contendo chumbo, metal extremamente tóxico, para fazer a soldagem de componentes eletrônicos em PCI $[2, \underline{3}]$. Diante das novas legislações, a indústria eletrônica tem procurado alternativas de eliminar o chumbo dos processos de soldagem $[4,5]$.

Os adesivos condutivos são compósitos constituídos por partículas metálicas, denominadas "fillers", dispersas em uma matriz polimérica. Dependendo da concentração de "fillers" os adesivos podem ser isotrópicos (denominados ICA) e conduzir corrente elétrica em todas as direções, ou serem anisotrópicos (denominados ACA) e ter um sentido preferencial de condução [ $[\underline{6}, 7]$.

Atualmente, a maioria dos adesivos condutivos isotrópicos comerciais são constituídos por uma mistura de pó e "flakes" de prata metálica dispersos em uma matriz de resina epóxi. A prata é utilizada devido a sua alta condutividade elétrica $\left(10^{7} \Omega^{-1} \mathrm{~m}^{-1}\right)$, maleabilidade e facilidade no processamento de partículas com diferentes formas. A resina epóxi é utilizada devido a seu fácil uso e disponibilidade de centenas de combinações de resina/endurecedor, que gera uma aderência geralmente superior. Essa combinação promove adesivos condutivos bastante funcionais.

Quando comparados com as pastas de solda, os ICAs apresentam uma série de vantagens, como por exemplo, capacidade de condução elétrica em pequenas áreas (fine-pitch), flexibilidade nas juntas de adesão, eliminação do uso de fluxo e temperaturas de trabalho mais baixas que no processo de soldagem convencional $[\underline{8}, \underline{9}, \underline{10}]$. Por outro lado, alguns problemas relacionados à fragilidade da junta adesiva e ao fato da condutividade elétrica ser ligeiramente mais baixa se comparado às soldas convencionais, seja ela "lead-free" ou estanho-chumbo ( $\mathrm{Sn}-\mathrm{Pb}$ ), são problemas que afetam a confiabilidade de componentes montados com adesivos condutivos.

O objetivo deste trabalho foi estudar as propriedades químicas e físicas, bem como o comportamento dos adesivos condutivos isotrópicos comerciais (forma de pasta) quando utilizados na montagem de componentes eletrônicos em placas de circuito impresso. A viabilidade, vantagens e desvantagens da utilização desses adesivos em todo processo de montagem SMT são discutidas e apresentadas.

\section{MATERIAIS E MÉTODOS}

Para o desenvolvimento deste trabalho, foram selecionados adesivos condutivos isotrópicos comerciais (na forma de pasta) de três diferentes fabricantes, sendo denominados neste trabalho como adesivos A, B e C. As especificações dos adesivos são apresentadas na Tabela 1. Contudo, para efeito de estudar o comportamento dos adesivos, algumas temperaturas utilizadas nos experimentos ultrapassaram as temperaturas recomendadas.

Inicialmente, os ICAs foram utilizados para preparar filmes espessos em substratos de vidro, com a finalidade de estudar as variações na resistividade elétrica de cada material em função da temperatura e do tempo de cura. Os ICAs foram depositados nos substratos utilizando tecnologia "screen-printer". Após a deposição, os filmes foram curados em estufa a $150^{\circ} \mathrm{C}$, temperatura recomendada pelos fabricantes dos adesivos A e C. Em intervalos de tempos regulares (10 min.), foram feitas medidas de resistividade elétrica das amostras utilizando o método de 4 pontas (equipamento Thin Film Devices - mod. FPP-2000). Também foram feitas medidas de resistividade elétrica em função da temperatura de cura. Neste caso, novas amostras foram preparadas. Variou-se a temperatura de cura entre 125 a $175^{\circ} \mathrm{C}$ e o tempo de cura foi mantido em 30 minutos.

Em uma segunda etapa, os ICAs foram utilizados para fazer a fixação de componentes eletrônicos (capacitores, resistores e semicondutores com terminal J) em placas "dummy" (Topline), utilizando a tecnologia de montagem em superfície (SMT). Após a montagem dos componentes sobre a placa, os adesivos foram curados a $150{ }^{\circ} \mathrm{C}$ por 40 minutos. Em seguida, foram preparadas amostras para análise microestrutural da interface placa/adesivo/componente. As amostras foram cortadas, embutidas em resina epóxi, polidas e caracterizadas por microscopia óptica (Microscópio Olympus SZX ILLD2-100 estéreo e Olympus PME metalográfico) e microscopia eletrônica de varredura.

Para fazer os testes elétricos funcionais, foram utilizadas placas de circuito impresso de LCD (mostrador de cristal líquido). Inicialmente, escolheu-se 5 resistores ôhmicos soldados com pasta de solda convencional e mediu-se a resistência, corrente elétrica e a tensão elétrica em cada um deles, utilizando um 
multímetro HP 34401A. Após essa etapa, os componentes foram removidos com o auxílio de uma estação de dessolda (PACE), limpos e fixados novamente com os adesivos condutivos isotrópicos. Então, foram medidas as mesmas propriedades e os resultados foram comparados e discutidos.

Tabela 1: Dados fornecidos pelos fabricantes dos ICAs utilizados neste trabalho

\begin{tabular}{|l|c|c|c|}
\cline { 2 - 4 } \multicolumn{1}{c|}{} & Adesivo A & Adesivo B & Adesivo C \\
\hline Fabricante/Marca & $\begin{array}{c}\text { Henkel } \\
\text { Loctite } 3880\end{array}$ & $\begin{array}{c}\text { Oxychem } \\
\text { Oxysim 502-40 }\end{array}$ & $\begin{array}{c}\text { Ablestik } \\
\text { Ablebond 84-1LMI }\end{array}$ \\
\hline Material Condutivo & Prata $(\mathrm{Ag})$ & Prata $(\mathrm{Ag})$ & Prata $(\mathrm{Ag})$ \\
\hline Tipo de Resina & Epóxi & Epóxi & Epóxi \\
\hline Viscosidade(1) & $7,5 \times 10^{4}-2,0 \times 10^{5} \mathrm{cP}$ & $3,5 \times 10^{4}-4,5 \times 10^{4} \mathrm{cP}$ & $2,2 \times 10^{4} \mathrm{cP}$ \\
\hline Tempo de Cura & $6 \min (2)$ & $15 \min (3)$ & $60 \min (2)$ \\
\hline $\begin{array}{l}\text { Temperatura de } \\
\text { Transição Vítrea }\end{array}$ & $40^{\circ} \mathrm{C}$ & $85^{\circ} \mathrm{C}$ & $103^{\circ}$ \\
\hline Resistividade(4) & $8,0 \times 10^{-4} \Omega \cdot \mathrm{cm}$ & $5,0 \times 10^{-1} \Omega \cdot \mathrm{cm}$ & $6,0 \times 10^{-4} \Omega \cdot \mathrm{cm}$ \\
\hline
\end{tabular}

1 - à $25^{\circ} \mathrm{C}$ e $5 \mathrm{rpm}$

2- à $150^{\circ} \mathrm{C}$

3 - à $120^{\circ} \mathrm{C}$

4- cada fabricante utiliza métodos diferentes para medida de resistividade

\section{RESULTADOS E DISCUSSÕES}

Na Figura 1 são apresentados os gráficos de resistividade elétrica em função da temperatura de cura obtidos para os adesivos estudados. O tempo de cura foi fixado em 30 minutos. Como pode ser observado nos gráficos da Figura 1, um aumento na temperatura de $150^{\circ} \mathrm{C}$ para $175^{\circ} \mathrm{C}$ praticamente não altera o valor da resistividade elétrica $\rho$ para as amostras A e C. Por isso, a temperatura de $150^{\circ} \mathrm{C}$ foi considerada como temperatura ideal de cura e foi utilizada nos demais testes deste trabalho. A amostra B teve um comportamento diferente das demais, pois a resistividade aumentou com o aumento da temperatura de cura (Figura 1).

Os gráficos de resistividade elétrica em função do tempo de cura obtidos para os filmes espessos dos adesivos são apresentados na Figura 2. Nestes experimentos a temperatura de cura foi fixada em $150^{\circ} \mathrm{C}$. Observa-se que os valores de resistividade permanecem praticamente constante após 30 min de cura para as amostras A e C. Para o adesivo B, a resistividade aumentou com o aumento do tempo de cura.

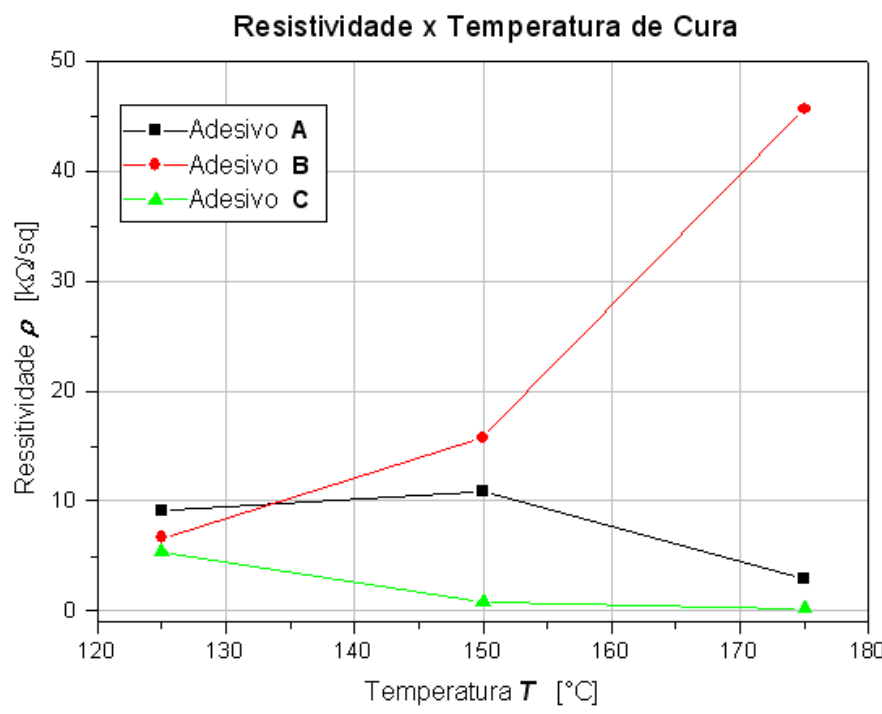

Figura 1: Gráficos de Resistividade versus Temperatura de Cura obtidos para os ICAs depositados em substratos de vidro e curados em estufa por $30 \mathrm{~min}$, em diferentes temperaturas. 
O comportamento apresentado pelo adesivo B (Figuras 1 e 2) pode estar relacionado com a degradação do polímero durante o processo de cura devido as altas temperaturas utilizadas. No entanto, novos estudos estão sendo realizados visando à comprovação desta hipótese.

Nas Figuras 3 a 5 são apresentadas às imagens obtidas por microscopia óptica da seção transversal das amostras preparadas com os diferentes ICAs e curadas a $150^{\circ} \mathrm{C}$ por 40 minutos. Em todos os casos é possível observar a boa adesão dos componentes às placas "dummy", indicando que é possível fazer a fixação de componentes eletrônicos em PCIs com adesivos condutivos. Nas micrografias obtidas por microscópio metalográfico (Figuras 3(b), 4(b) e 5(b)) é possível observar a presença dos "fillers" por toda a extensão da junta. Também pode se observar que há uma maior deposição dos "fillers" próxima às interfaces pad/adesivo e componente/adesivo. A espessura da camada de "fillers" nestas interfaces pode chegar até aproximadamente $8 \mu \mathrm{m}$ em espessura.

Comparando as Figuras 3(b), 4(b) e 5(b), nota-se que a quantidade de "fillers" no adesivo B é bem menor que nos demais. Isto comprova os altos valores de resistividade obtidos para este adesivo.

Na Figura 6 está apresentada à micrografia obtida por MEV da junção placa/adesivo/componente. Observa-se que não há formações de camadas intermetálicas, como ocorre no processo de solda convencional.

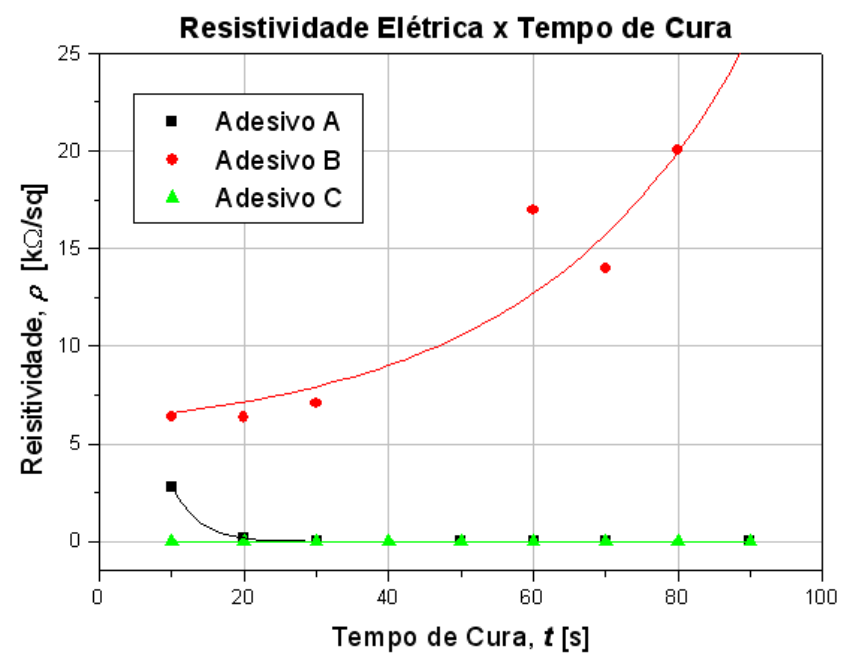

Figura 2: Gráfico Resistividade versus Tempo de Cura para temperatura de $150{ }^{\circ} \mathrm{C}$. Adesivos A, B, C

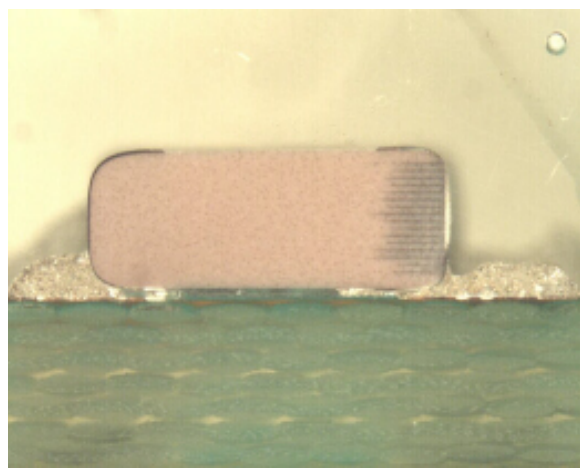

(a)

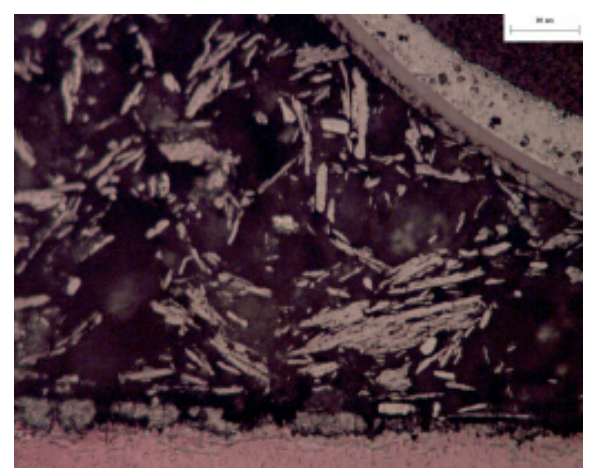

(b)

Figura 3: Resistor fixado com Adesivo A. (a) foto obtida com estéreo microscópio - ampliação de 32x. (b) micrografia obtida com microscópio metalográfico - ampliação de 400x. 


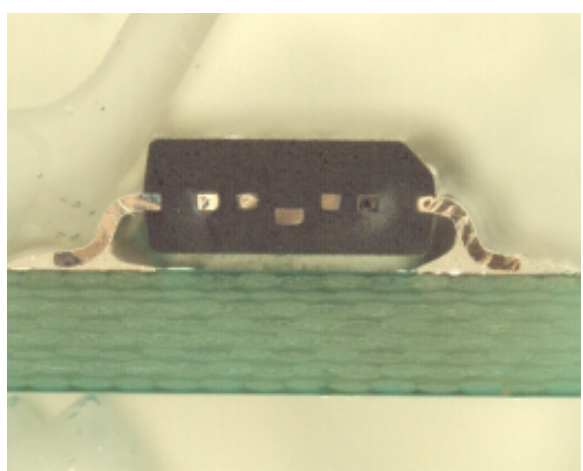

(a)

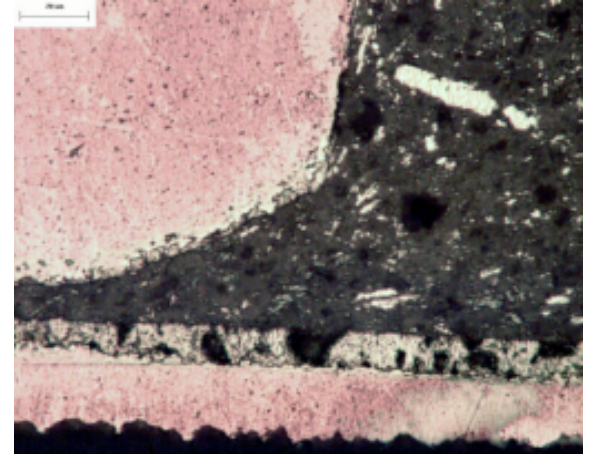

(b)

Figura 4: Encapsulamento SOIC fixado com Adesivo B. (a) foto obtida com estéreo microscópio ampliação de 32x. (b) micrografia obtida com microscópio metalográfico - ampliação de 400x.

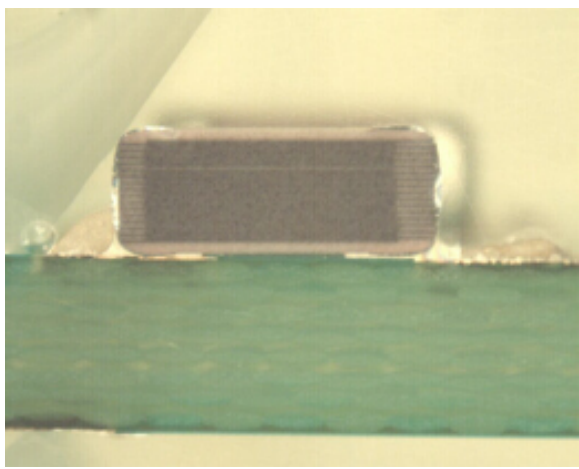

(a)

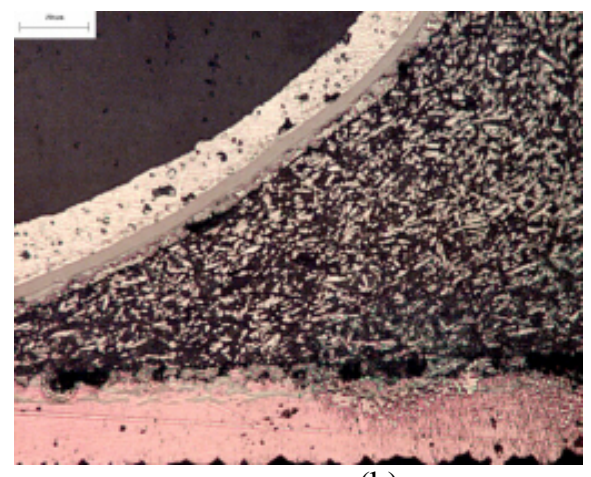

(b)

Figura 5: Resistor fixado com Adesivo C. (a) foto obtida com estéreo microscópio - ampliação de 32x. (b) micrografia obtida com microscópio metalográfico - ampliação de 400x.

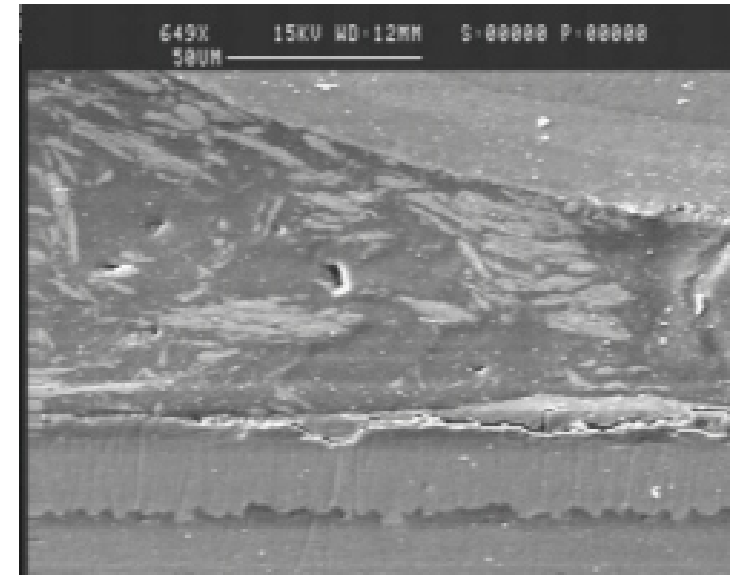

Figura 6: Micrografia obtida por MEV da junção placa/adesivo/componente da amostra preparada com o Adesivo A.

Na Tabela 2 estão apresentados os resultados das medidas de resistência, tensão e corrente elétrica obtidos nos testes elétricos funcionais realizados em placas de circuitos de LCD, tanto com componentes fixados com solda $\mathrm{Sn}-\mathrm{Pb}$ quanto com adesivos condutivos, bem como a diferença em porcentagem entre as duas medidas. 
É possível observar que o valor da diferença relativa das medidas dos resistores R1, R2, R4 e R5 (resistores com valor nominal igual a $2,2 \mathrm{k} \Omega$ ) tiveram variações menores que $\pm 10 \%$. Já o resistor R3 (valor nominal igual a $20 \mathrm{k} \Omega$ ) teve uma diminuição do valor da resistência elétrica em torno de $35 \%$. Contudo, a variação dos demais parâmetros não ultrapassou $2,1 \%$, não alterando as características do circuito como um todo. Na Figura 7 são apresentadas as fotos obtidas dos displays em funcionamento, com componentes fixados com adesivos condutivos. Isto comprova a viabilidade de se utilizar os ICAs como alternativas as soldas nos processos de montagem de componentes eletrônicos em tecnologia de montagem em superfície. As vantagens do uso dos ICAs estudados são a menor temperatura de cura $\left(150{ }^{\circ} \mathrm{C}\right)$, quando comparada às soldas $\mathrm{Sn}-\mathrm{Pb}$ (temperatura de fusão $183{ }^{\circ} \mathrm{C}$ ) e soldas livres de chumbo $\left(233{ }^{\circ} \mathrm{C}\right)$, diminuindo os riscos de degradação dos componentes eletrônicos e das PCIs devido altas temperaturas; a possibilidade de utilizar os mesmos equipamentos do processo atual de montagem de componentes eletrônicos e o menor custo, quando comparados às soldas livres de chumbo.

Tabela 2: Valores Obtidos no teste elétrico

\begin{tabular}{|c|c|c|c|c|c|c|c|c|c|c|c|}
\hline & \multirow[b]{2}{*}{ Componente } & \multirow[b]{2}{*}{ Valor nominal } & \multicolumn{3}{|c|}{ Solda } & \multicolumn{3}{|c|}{ Adesivo } & \multicolumn{3}{|c|}{ Diferença } \\
\hline & & & Resistência $(k \Omega)$ & $\overline{\text { Tensão (V) }}$ & Corrente $(\mathrm{mA})$ & Resistência $(k \Omega)$ & Tensão (V) & Corrente $(\mathrm{mA})$ & Resistência (\%) & Tensão (\%) & Corrente (\%) \\
\hline \multirow{3}{*}{ PLACA A } & R2 & $2,2 \mathrm{k} \Omega$ & 2,193 & 1,757 & 0,860 & 2,162 & 1,762 & 0,856 & $-1,4 \%$ & $0,3 \%$ & $-0,47 \%$ \\
\hline & R4 & $2,2 \mathrm{k} \Omega$ & 2,191 & 1,758 & 0,862 & 2,197 & 1,761 & 0,863 & $0,3 \%$ & $0,2 \%$ & $0,12 \%$ \\
\hline & R5 & $2,2 \mathrm{k} \Omega$ & 2,175 & 1,774 & 0,873 & 2,185 & 1,767 & 0,874 & $0,5 \%$ & $-0,4 \%$ & $0,11 \%$ \\
\hline \multirow{3}{*}{ PLACA B } & $\mathrm{R} 1$ & $2,2 \mathrm{k} \Omega$ & 2,223 & 1,792 & 0,878 & 2,411 & 1,831 & 0,875 & $8,5 \%$ & $2,2 \%$ & $-0,34 \%$ \\
\hline & R4 & $2,2 \mathrm{k} \Omega$ & 2,203 & 1,783 & 0,869 & 2,259 & 1,827 & 0,865 & $2,5 \%$ & $2,5 \%$ & $-0,46 \%$ \\
\hline & R5 & $2,2 \mathrm{k} \Omega$ & 2,211 & 1,778 & 0,879 & 2,302 & 1,800 & 0,877 & $4,1 \%$ & $1,2 \%$ & $-0,23 \%$ \\
\hline \multirow[b]{3}{*}{ PLACA C } & R1 & $2,2 \mathrm{k} \Omega$ & 2,218 & 1,780 & 0,827 & 2,219 & 1,780 & 0,871 & $0,0 \%$ & $0,0 \%$ & $5,32 \%$ \\
\hline & R2 & $2,2 \mathrm{k} \Omega$ & 2,196 & 1,763 & 0,863 & 2,203 & 1,755 & 0,857 & $0,3 \%$ & $-0,5 \%$ & $-0,70 \%$ \\
\hline & R3 & $20 \mathrm{k} \Omega$ & 17,450 & 15,963 & 2,565 & 11,926 & 15,927 & 2,559 & $-31,7 \%$ & $-0,2 \%$ & $-0,23 \%$ \\
\hline
\end{tabular}

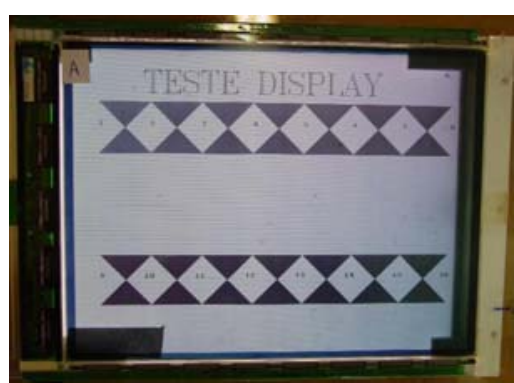

(a)

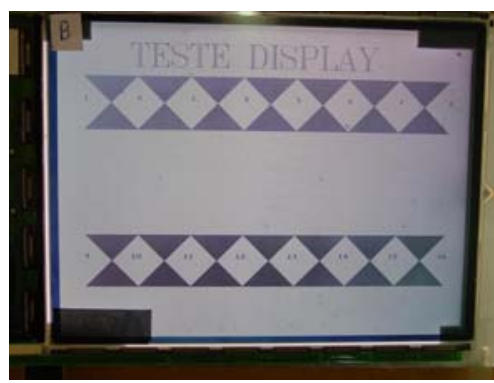

(b)

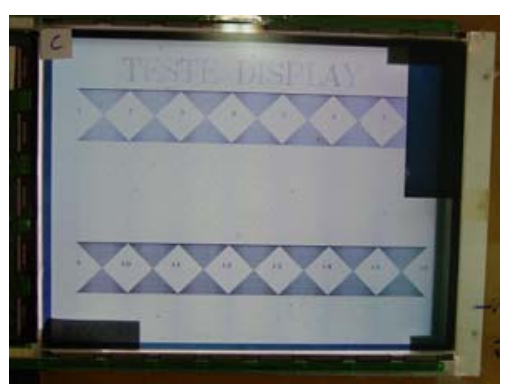

(c)

Figura 7: Imagens obtidas da aparência dos displays testados. Figuras (a), (b) e (c) com componentes fixados com adesivos condutivos $\mathrm{A}, \mathrm{B}$ e $\mathrm{C}$, respectivamente.

\section{CONCLUSÕES}

Os estudos do uso de adesivos condutivos isotrópicos na montagem de componentes eletrônicos em PCIs com tecnologia SMT ainda estão em fase inicial. Contudo, os resultados obtidos apontam que o uso destes adesivos é bastante viável. O desempenho dos displays não se alterou quando os componentes fixados com pasta de solda foram removidos e fixados novamente com os adesivos condutivos isotrópicos (na forma de pasta). Contudo, para determinados tipos de circuitos, é necessário levar em conta as diferenças dos valores entre as pastas de solda e os adesivos no momento do projeto.

As temperaturas de cura dos adesivos estudados são mais baixas quando comparadas às temperaturas de fusão das pastas de solda e, portanto, são menos agressivas para os componentes e placas de circuito impresso, aumentando assim a janela do processo. 
Com relação à fragilidade da junta adesiva, se fazem necessários ensaios mecânicos e testes de confiabilidade a fim de estimar a vida útil de produtos eletrônicos montados com esse tipo de material.

\section{BIBLIOGRAFIA}

[1] RoHS, http://www.rohs.gov.uk. Acessado em junho de 2005.

[2] YOON, J.W., KIM, S. W., JUNG, S.B., "IMC Growth and Shear Strength of Sn-Ag-Bi-In/Au/Ni/Cu BGA Joints During Aging”, Materials Transactions, v. 45, n. 3, pp. 727-733, 2004

[3] SUGANUMA, K., “Advances in lead-free Electronics Soldering”, Current Opinion Solid State Materials Science, v. 5, n. 1, pp. 55-64, 2001.

[4] HUIT, I.K., RALPH, B., "A Study of the Initiation of the Tombstoning Effect on Leadless Chips", International Journal of Machine Tools and Manufacture, v. 35, n. 9, p. 1251-1268, 1995.

[5] CANNIS, J., “Green IC Packaging”, Advanced Packaging, p. 33, August 2001.

[6] LIONG, S., WONG, C.P., BURGOYNE JR., W.F., "Conductivity Improvement of Thermoplastic Isotropically Conductive Adhesives", In: Proceedings of Electronic Components and Technology Conference, pp. 1140-1146, 2002

[7] LIONG, S., WONG, C.P., BURGOYNE JR., W.F., "Adhesion Improvement of Thermoplastic Isotropically Conductive Adhesives", In: Proceedings of Electronic Components and Technology Conference, pp. 1631-1639, 2002

[8] PÉRICHAUD, M.G., DELÉTAGE, J.Y., FRÉMONT, H., et al., "Evaluation of Conductive Adhesives for Industrial SMT Assemblies", In: Proceedings of International Electronics Manufacturing Technology Symposium, pp. 377 - 385, 1998

[9] INADA, T., WONG, C.P., "Fundamental Study on Adhesive Strength of Electrical Conductive Adhesive (ECAs)", In: Proceedings of Conference on Adhesive Joining Technology in Electronics, pp. 156159,1998

[10] FUJimOtO, F., MAZON , T., SCHENKEL, E. A., BIASOLI, M. T., “Conductive Adhesives: To use or not?", In: Proceedings of the IMAPS Brazil, cd-rom, Brasil, 2005 